\title{
EDITORIAL
}

\section{A kinder, gentler way: control of the proliferative tumor compartment, not cosmetic complete response, should be the goal of myeloma therapy}

\section{Leukemia (2008) 22, 673-675; doi:10.1038/leu.2008.2}

In this issue of Leukemia, Arzoumanian et al. ${ }^{1}$ advocate a new clinical end point for multiple myeloma (MM) therapy: suppression of the abnormal karyotype. It is an impressive summary of the analysis of metaphase cytogenetics in 2000 patients with MM treated at the University of Arkansas for Medical Sciences. It establishes a new paradigm for evaluating relapsed MM and clearly demonstrates that relapsed MM may be effectively divided into different groups with markedly different outcomes, in much the same way that untreated MM has been divided into different molecular ${ }^{2,3}$ and prognostic groups. ${ }^{4}$ To put their findings in context, it is helpful to review the natural history of plasma cell neoplasia.

Multiple myeloma is characterized by the progressive accumulation of malignant plasma cells in the bone marrow causing anemia and bone destruction. It is often preceded by a stable and limited monoclonal expansion of benign plasma cells in the bone marrow that do not cause any end-organ damage. In examining isolated plasma cells, we are unable to identify genetic or gene expression features that distinguish malignant from benign monoclonal gammopathy. In practice, therefore, a combination of clinical features, including temporal stability, minimal plasmacytosis (less than $10 \%$ of bone marrow mononuclear cells) and lack of end organ damage, is used to identify benign monoclonal gammopathy. This condition has been variously termed 'benign monoclonal gammopathy', 'monoclonal gammopathy of unknown etiology', 'essential hyperglobulinemia' and 'dysimmunoglobulinemia', ${ }^{5-8}$ although the generally accepted term is now 'monoclonal gammopathy of undetermined significance' ${ }^{9}$ This latter, rather awkward historical term derives from a time when the natural history of this condition was not known, and reflects the inability to distinguish either pathologically or molecularly, benign from malignant plasma cells. In patients in whom MM is relapsing from a (complete) remission, we are again faced with the dilemma of distinguishing benign (or more appropriately, minimally malignant) from aggressively malignant plasma cells.

In contrast to the situation for relapsed $\mathrm{MM}$, a great deal of effort has been focused on stratifying untreated $M M$ into different groups with more uniform outcomes. These include staging systems (Durie-Salmon and International Staging System) that quantitate tumor mass (level of monoclonal gammopathy and $\beta 2$-microglobulin) and extent of host endorgan damage (lytic bone lesions, anemia and hypoalbuminemia) as a measure of tumor burden and aggressivity. ${ }^{10,11}$ More powerful are classifications based on genetics, which seek to identify different molecular subtypes of MM. Although primarily designed based on underlying genetics, these classification systems also have important prognostic implications. The genetics of $\mathrm{MM}$ has been divided into primary and secondary events. The primary events are thought to be immunoglobulin gene translocations involving 4p16 (FGFR3/MMSET), 16q23 \& $20 q 11 \& 8 q 24$ (c-maf, mafB and mafA), 6p21 \& 11q13 (cyclin
D3 and cyclin D1), and hyperdiploidy (mainly trisomies of chromosomes $3,5,7,9,11,15,19$ and 21). ${ }^{12}$ Of these, the 4 p16 and 16q23 translocations have a poor prognosis (that we expect is also shared by the mafA and mafB translocations). Secondary genetic events include activating mutations of ras, translocations of MYC, deletion of chromosome 13 and deletion of p53, with the latter two associated with a poor prognosis. Translocations of $4 p 16$ and $16 q 23$ and deletion of p53 are strongly associated with deletion of chromosome 13 (>78\% of cases), so that in a multivariate analysis including all these genetic abnormalities, deletion of chromosome 13 was not identified as an independent prognostic factor. ${ }^{13}$ However, genetic factors alone do not allow sufficient discrimination, and in a large study of 1000 patients from the Intergroupe Francaise du Myelome, it was found that the addition of $\beta 2$-microglobulin to $t(4 ; 14)$ and deletion of p53 provided the most robust prognostic model. ${ }^{13}$

Finally, the addition of a measure of plasma cell proliferation provides a final independent prognostic factor. This can be done prospectively on bone marrow aspirates using 5-bromo-2deoxyuridine incorporation in vitro (the Plasma Cell Labeling Index (PCLI), with a cutoff of $1 \%$ ), and as with cytogenetic abnormality (CA), an elevated PCLI in patients in plateau phase $\mathrm{MM}$ is associated with a poor prognosis. ${ }^{14}$ More recently, proliferation has been measured retrospectively on bone marrow biopsies using a double immunohistochemical stain for nuclear Ki67 and surface CD138 (with a cutoff of 4\%). ${ }^{15}$ Not surprisingly, an elevated Ki67 correlated with the presence of abnormal metaphase karyotype (CA) (82\% of patients with an elevated $\mathrm{Ki} 67$ index vs $43 \%$ in the remaining patients), furthermore, Ki67 index was elevated in 93\% of the patients with hypodiploidy, whereas no correlation was found with hyperdiploidy. In view of recent studies using array-based comparative genomic hybridization, it is clear that almost all MM patients have genetic changes that should be evident with conventional cytogenetics. ${ }^{16,17}$ The failure to detect metaphase karyotype abnormalities in an MM patient does not mean the MM tumor is cytogenetically normal, but is attributed to a failure to stimulate the MM cells to divide in vitro, and provide appropriate metaphase nuclei for analysis. Therefore, the detection of $\mathrm{CA}$ in relapsing $\mathrm{MM}$ patients by Arzoumanian et al. is a measure of both the MM tumor burden and their intrinsic proliferative ability, perhaps accounting for its powerful prognostic impact. $^{1}$

This report from the Arkansas group is significant for what it does not say. It does not say that suppression of the myeloma clone is critical for long-term survival. Their conclusion is that it is sufficient simply to suppress abnormal karyotypes. After leading the field for the last two decades in the pursuit of ever-higher complete responses, this is a seemingly remarkable admission, although perfectly consistent with their recent publications and editorials. ${ }^{18,19}$ It is becoming increasingly clear that the qualitative impact of a complete response is dependent on the treatment employed, with the most significance given to complete responses following high-dose 




Figure 1 Percentage change in stock price of Celgene (CELG) and Millenium Pharmaceuticals (MLNM) around the time of the 2007 annual meeting of the American Society of Hematology (ASH).

melphalan, and the least significance to complete responses to glucocorticoids. There are insufficient data to conclude about the quality of complete responses to thalidomide, lenalidomide or bortezomib, although I am willing to hazard a guess that we will find thalidomide and lenalidomide somewhat closer to melphalan, and bortezomib closer to glucocorticoids. A lack of information has not prevented the field from single-mindedly pursuing the 'cosmetic' complete response, defined as one that makes the principal investigator look good, but does not provide meaningful benefit to the patient (and in fact may be harmful). The seductive allure of the cosmetic complete response propels the investigator's career, attracts patients to clinical trials, facilitates presentation and publication of clinical trials, helps pharmaceutical companies raise capital and compels stock analysts to trade pharmaceutical stocks. The irony of the situation was highlighted following the 2007 annual meeting of the American Society of Hematology.

Surrounding the time of this meeting, the price of Celgene (the manufacturer of thalidomide and lenalidomide) stock fell $30 \%$, and the price of Millenium Pharmaceuticals (the manufacturer of bortezomib) stock rose $50 \%$ (Figure 1). It is of course impossible to pinpoint all of the factors that play into the valuations of stocks, but it is notable that the sharp drop in Celgene stock followed the oral presentation ${ }^{20}$ of the results of the E4A03 study comparing lenalidomide with high or low-dose dexamethasone. The oral presentation contained response rate data not present in the published abstract including a complete response + very good partial response (CR + VGPR) after four cycles of 46 vs $26 \% \quad(P<0.001)$, that was presumably disappointingly low for some analysts, despite the fact that there is a significant difference in the 2-year probability of survival of 75 vs $87 \%(P<0.009)$. The obvious conclusion is that response rate after four cycles is not a good surrogate for longterm survival, although this view is apparently not rewarded by the financial markets. In contrast, Jesus San Miguel ${ }^{21}$ presented the results of the VISTA trial showing significantly better response rates, progression-free survival and overall survival with melphalan, prednisone and bortezomib treatment compared to melphalan and prednisone treatment. This was accompanied by a complete response rate of close to $30 \%$ and is very encouraging, although one could argue that the dramatic increase in the complete response was not accompanied by an equally dramatic prolongation of overall survival.

Hopefully the results of these and other clinical trials, together with the careful analysis of patient outcomes studied with correlative studies similar to those described by Arzoumanian et al. will temper the thoughtless enthusiasm for cosmetic complete response, and help to identify other valuable surrogates of long-term disease survival, such as the suppression of cytogenetic abnormalities as reported here.

PL Bergsagel

Department of Medicine, Mayo Clinic College of Medicine, 13400 E Shea Blvd., Scottsdale, AZ, USA E-mail: bergsagel.leif@mayo.edu

\section{References}

1 Arzoumanian V, Hoering A, Sawyer J, van Rhee F, Bailey C, Gurley J et al. Suppression of abnormal karyotypes is critical for prolonged survival in multiple myeloma. Leukemia 2008 (in press).

2 Bergsagel PL, Kuehl WM, Zhan F, Sawyer J, Barlogie B, Shaughnessy Jr J. Cyclin D dysregulation: an early and unifying pathogenic event in multiple myeloma. Blood 2005; 106: 296-303.

3 Zhan F, Huang Y, Colla S, Stewart JP, Hanamura I, Gupta S et al. The molecular classification of multiple myeloma. Blood 2006; 108: 2020-2028.

4 Shaughnessy Jr JD, Zhan F, Burington BE, Huang Y, Colla S, Hanamura I et al. A validated gene expression model of high-risk multiple myeloma is defined by deregulated expression of genes mapping to chromosome 1. Blood 2006; 109: 2276-2284.

5 Waldenstrom J. Studies on conditions associated with disturbed gamma globulin formation (gammopathies). Harvey Lect 1960; 56: 211-231.

6 Osserman EF, Takatsuki K. Plasma cell myeloma: gamma globulin synthesis and structure. A review of biochemical and clinical data, 
with the description of a newly-recognized and related syndrome, 'H-Gamma-2-chain (Franklin's) disease. Medicine (Baltimore) 1963; 42: 357-384.

7 Waldenstrom J. The occurrence of benign, essential monoclonal (M Type), non-macromolecular hyperglobulinemia and its differential diagnosis. IV. Studies in the gammapathies. Acta Med Scand 1964; 176: 345-365.

8 Zawadzki ZA, Edwards GA. Dysimmunoglobulinemia in the absence of clinical features of multiple myeloma and macroglobulinemia. Am J Med 1967; 42: 67-88.

9 Kyle RA, Therneau TM, Rajkumar SV, Offord JR, Larson DR, Plevak MF et al. A long-term study of prognosis in monoclonal gammopathy of undetermined significance. N Engl J Med 2002 346: $564-569$.

10 Durie BG, Salmon SE. A clinical staging system for multiple myeloma. Correlation of measured myeloma cell mass with presenting clinical features, response to treatment, and survival. Cancer 1975; 36: 842-854.

11 Greipp PR, San Miguel J, Durie BG, Crowley JJ, Barlogie B, Blade J et al. International staging system for multiple myeloma. J Clin Oncol 2005; 23: 3412-3420.

12 Kuehl WM, Bergsagel PL. Multiple myeloma: evolving genetic events and host interactions. Nat Rev Cancer 2002; 2: 175-187.

13 Avet-Loiseau H, Attal M, Moreau P, Charbonnel C, Garban F, Hulin $\mathrm{C}$ et al. Genetic abnormalities and survival in multiple myeloma: the experience of the Intergroupe Francophone du Myelome. Blood 2007; 109: 3489-3495.

14 Steensma DP, Gertz MA, Greipp PR, Kyle RA, Lacy MQ, Lust JA et al. A high bone marrow plasma cell labeling index in stable plateau-phase multiple myeloma is a marker for early disease progression and death. Blood 2001; 97: 2522-2523.

15 Gastinne T, Leleu X, Duhamel A, Moreau AS, Franck G, Andrieux J et al. Plasma cell growth fraction using $\mathrm{Ki}-67$ antigen expression identifies a subgroup of multiple myeloma patients displaying short survival within the ISS stage I. Eur J Haematol 2007; 79: 297-304.

16 Carrasco DR, Tonon G, Huang Y, Zhang Y, Sinha R, Feng B et al. High-resolution genomic profiles define distinct clinicopathogenetic subgroups of multiple myeloma patients. Cancer Cell 2006; 9: 313-325.

17 Keats JJ, Fonseca R, Chesi M, Schop R, Baker A, Chng WJ et al. Promiscuous mutations activate the noncanonical NF-kappaB pathway in multiple myeloma. Cancer Cell 2007; 12: 131-144.

18 Barlogie B, Tricot G. Complete response in myeloma: a Trojan horse? Blood 2006; 108: 2134.

19 Haessler J, Shaughnessy Jr JD, Zhan F, Crowley J, Epstein J, van Rhee $\mathrm{F}$ et al. Benefit of complete response in multiple myeloma limited to high-risk subgroup identified by gene expression profiling. Clin Cancer Res 2007; 13: 7073-7079.

20 Rajkumar SV, Jacobus S, Callander N, Fonseca R, Vesole D, Williams $\mathrm{M}$ et al. A randomized trial of lenalidomide plus highdose dexamethasone (RD) versus lenalidomide plus low-dose dexamethasone $(\mathrm{Rd})$ in newly diagnosed multiple myeloma (E4A03): A trial coordinated by the eastern cooperative oncology group. Blood (ASH Annual Meeting Abstracts) 2007; 110: 74.

21 San Miguel JF, Schlag R, Khuageva N, Shpilberg O, Dimopoulos M, Kropff $M$ et al. MMY-3002: a phase 3 study comparing bortezomib-melphalan-prednisone (VMP) with melphalanprednisone (MP) in newly diagnosed multiple myeloma. Blood (ASH Annual Meeting Abstracts). 2007; 110: 76. 